\author{
Roberto POGGI*
}

\title{
Presenza in Italia di Alfieriella Wittmer, 1935 con dati sulle specie attribuite al genere (Coleoptera Cryptophagidae)
}

\begin{abstract}
Riassunto: Si rendono note le località italiane di raccolta di Alfieriella naxiana (Reitter, 1884), che figurava già citata per l'Italia, ma senza dati precisi. Con l'occasione si presenta una piccola revisione di tutte e tre le specie del genere rinvenibili lungo le coste del Mediterraneo centro-orientale, illustrando in particolare i caratteri edeagici che permettono una sicura identificazione dei taxa.
\end{abstract}

\begin{abstract}
Presence in Italy of Alfieriella Wittmer, 1935 with data on the species attributed to the genus (Coleoptera Cryptophagidae).
The Italian records of Alfieriella naxiana (Reitter, 1884) are listed; the species was already mentioned for Italy, but without precise data. With the occasion, a short revision of the three species of the genus living along the central-eastern Mediterranean coasts is presented, illustrating in particular the aedeagic characters that allow a sure identification of the taxa.
\end{abstract}

Key words: Cryptophagidae, Alfieriella, Mediterranean species, faunistic records.

\section{PREMESSA}

Johnson et al. (2007) citano tra i Cryptophagidae presenti sul territorio italiano anche Alfieriella naxiana (Reitter, 1884), che pochi anni dopo Otero (2013) segnala più dettagliatamente per l'Italia e la Sicilia. I dati derivano da informazioni trasmesse a José Carlos Otero dal comune amico Fernando Angelini, sulla base di materiali da tempo identificati da me, ma mai formalmente pubblicati e per questo non riportati in Angelini (1995). Con questo contributo mi propongo di colmare questa lacuna.

\section{GLI ANTECEDENTI}

Una trentina d'anni fa Alessandro Focarile mi inviò in esame un esemplare di Cryptophagidae da lui raccolto a Carlentini, in Sicilia, che non riusciva ad associare a nessuna delle specie segnalate per l'Italia. Approfondendo le ricerche, trovai con sorpresa un individuo simile presente in collezione Dodero, raccolto dallo stesso Dodero nel 1913 in un'area abbastanza prossima alla precedente (a Pachino) e da lui identificato come "Cyprogenia naxiana var.", ma la cui esistenza non era mai stata resa nota.

In seguito, in un invio di Coleotteri dell'Aspromonte (Calabria) sottopostomi in studio da Fernando Angelini, rinvenni una serie di esemplari identici a quelli siciliani, che inizialmente ipotizzai potessero ap- partenere ad una forma inedita. Comunque, per definire con un margine di accettabile sicurezza il nome da attribuire ai suddetti individui, chiesi ed ottenni in visione da diversi musei, grazie soprattutto alla collaborazione di Otero, esemplari tipici dei taxa più prossimi a quello in esame. La conclusione cui pervenni fu che gli individui italiani potevano identificarsi effettivamente come Alfieriella naxiana (Reitter, 1884), confermando così l'ipotesi di Dodero. La specie è stata in seguito raccolta abbondantemente e a più riprese da Angelini in Aspromonte e in Sicilia sudorientale.

\section{IL GENERE ALFIERIELLA WITTMER, 1935}

Il genere Alfieriella è stato compiutamente trattato dal punto di vista morfologico e sistematico da Ratti (1976) (a cui rimando per gli approfondimenti), il quale ha dimostrato che le specie tipiche rispettivamente di Cyprogenia Baudi di Selve, 1870 (C. denticulata Baudi di Selve, 1870) e di Alfieriella Wittmer, 1935 (A. rabinovitchi Wittmer, 1935) sono congeneri, ma il nome valido per indicare il genere è quello proposto da Wittmer, perché il nome scelto da Baudi risulta preoccupato da Cyprogenia Agassiz, 1852 (Mollusca, Unionidae). Strand (1936), accortosi dell'esistenza dell'omonimia, aveva proposto per Cyprogenia Baudi il nuovo nome Obenbergerodes, ma su di esso ha comunque precedenza cronologica, per un anno, Alfieriella.

${ }^{*}$ Roberto Poggi, Conservatore Onorario a vita, Museo Civico di Storia Naturale "G. Doria”, Via Brigata Liguria 9, 16121 Genova, Italia. E-mail: rpoggi@comune.genova.it 
Nel tempo la collocazione sistematica del genere è stata oggetto di diversi mutamenti; esso infatti, attribuito inizialmente ai Colydiidae, è poi passato ai Cucujidae per confluire nei Cryptophagidae.

E' comunque ormai acclarata la sua affinità con Hypocoprus Motschulsky, 1839, tanto che, confermando pienamente la prima ipotesi avanzata da Ganglbauer (1899: 845 e 897), adottata da Jakobson (1915) e Reitter (1922), poi compiutamente definita da Ratti (1976) e infine ripresa da Ślipiński (1983), Leschen (1996) ha inserito il genere, all'interno dei Cryptophagidae, nella sottofamiglia Atomariinae tribù Hypocoprini (in compagnia appunto di Hypocoprus nonché di Amydropa Reitter, 1877 e Hypophagus Liubarsky, 1989), seguito poi sia da Johnson et al. (2007) che da Bouchard et al. (2011).

Crowson (1980) ritenne invece di dover istituire per Alfieriella (e, con dubbio, per Amydropa) la sottofamiglia Alfieriellinae, che però rimase un nomen nudum, in quanto l'autore non ne fornì mai la diagnosi (ICZN art. 13.1).

Per completare il quadro generale, va segnalato che in Fauna Europaea (Otero, 2013) permane tuttora una situazione piuttosto ambigua, in quanto il genere Alfieriella è inserito nella tribù Hypocoprini della sottofamiglia Hypocoprinae, ma contemporaneamente in tale sottofamiglia figura anche la tribù Alfieriellini, senza rappresentanti, così come nell'ambito della famiglia Cryptophagidae è riportata la sottofamiglia Alfieriellinae, anch'essa senza rappresentanti.

L'inquadramento sistematico di Alfieriella nell'ambito delle sottofamiglie e tribù dei Cryptophagidae italiani si può evidenziare nel sintetico prospetto tabellare che segue, tratto ed adattato da Leschen (1996).

- Cavità procoxali posteriormente chiuse .Cryptophaginae

- Hypomeron del prosterno con incisione a lato dell'inserzione delle procoxae

Cryptophagini

[Con i generi: Telmatophilus Heer, Paramecosoma Curtis, Henoticus C.G. Thomson, Pteryngium Reitter, Micrambe C.G. Thomson, Cryptophagus Herbst, Spavius Motschulsky (= Emphylus Erichson) e Antherophagus Latreille].

- Hypomeron del prosterno senza incisione a lato dell'inserzione delle procoxae.......Caenoscelini (Con i generi: Caenoscelis C.G. Thomson e Sternodea Reitter).
- Cavità procoxali posteriormente aperte

Atomariinae

- Sutura fronto-clipeale presente; carena del pronoto presente Atomariini (Con i generi: Atomaria Stephens, Ootypus Ganglbauer, Curelius Casey ed Ephistemus Stephens).

- Sutura fronto-clipeale assente; carena del pronoto assente Hypocoprini

- Capo senza spina sottogenale; antenne di undici articoli, con clava di tre; elitre non ricoprenti interamente l'addome e con omeri non dentati; ali presenti; mesepimeron e mesosterno separati.

Hypocoprus Motschulsky

- Capo con spina sottogenale; antenne di dieci articoli, con clava di uno; elitre ricoprenti interamente l'addome e con omeri dentati; ali assenti; mesepimeron e mesosterno fusi ..... Alfieriella Wittmer

Ad Alfieriella sono attualmente attribuite quattro specie, con distribuzione compresa tra il Mediterraneo e l'Asia centrale. Johnson et al. (2007) citano infatti:

- denticulata (Baudi di Selve, 1870) di Cipro, Libano e Siria;

- laticollis (Reitter, 1891) di Uzbekistan;

- naxiana (Reitter, 1884) di Grecia e Italia;

- rabinovitchi Wittmer, 1935 di Egitto.

Ad esse va però aggiunta la n. sp. inedita di Deutang (Sikkim, India), conservata nel Natural History Museum, London, cui fanno cenno Ratti (1976) e Crowson (1980); di tale taxon, citato come nuovo genere e nuova specie (sub "Acryptus sikkimensis Sen Gupta, unpublished"), sono stati forniti i caratteri morfologici in una tesi di laurea (Behura, 1992) resa consultabile online nel 2018; Leschen (1996), che ha esaminato gli esemplari, ha già considerato il supposto nuovo genere Acryptus un sinonimo di Alfieriella.

Inoltre, secondo quanto comunicatomi anni fa da Enrico Ratti (in litt., 1986), un'ulteriore specie, proveniente dall'Afghanistan e verosimilmente inedita, è conservata nel Museo di Budapest.

Gli ultimi due taxa, così come Alfieriella laticollis (Reitter, 1891) di Tashkent (Uzbekistan), non sono stati presi in considerazione in questa sede. 
Dal punto di vista ecologico Leschen (1999) osserva che il genere Alfieriella, così come Amydropa Reitter, 1877 (suo "sister group" americano, con due specie di Baja California e Cile), è tendenzialmente legato ad ambienti piuttosto aridi, se non desertici.

\section{TRATTAZIONE DELLE SPECIE}

Con i seguenti acronimi si indicano le collezioni e i musei dove sono conservati gli esemplari controllati e citati nel corso del lavoro:

$\mathrm{CFA}=$ collezione Fernando Angelini, Francavilla Fontana, Brindisi;

CFA-MZUF $=$ collezione Fernando Angelini in Museo di Storia Naturale dell'Università di Firenze, Sezione di Zoologia "La Specola";

$\mathrm{HMNH}=$ Hungarian Museum of Natural History, Budapest (Ungheria);

MRSNT $=$ Museo Regionale di Scienze Naturali, Torino;

MSNG = Museo Civico di Storia Naturale "Giacomo Doria", Genova;

MSNM = Museo Civico di Storia Naturale, Milano;

NHMW $=$ Naturhistorisches Museum, Wien (Austria).

Le mie ricerche sono state possibili per la cordiale collaborazione ricevuta da tutti i colleghi ed amici che negli ultimi decenni si sono alternati nella responsabilità dei musei sopra elencati e a cui rivolgo un ringraziamento collettivo; sono poi grato a José Carlos Otero, Enrico Ratti, Constantinou Papaconstantinou e Marco Alberto Bologna per la collaborazione prestatami e a Fernando Angelini e Alessandro Focarile per il materiale fornitomi e per la pazienza dimostrata nei miei riguardi durante la lunga attesa della pubblicazione dei loro dati.

Alfieriella denticulata (Baudi di Selve, 1870)

(Figg. 1, 5, 16-17, 20)

Cyprogenia denticulata Baudi di Selve, 1870: 54; Reitter, 1882: 136; Reitter, 1884: 66; Reitter, 1891: 197; Jakobson, 1915: 906; Hetschko, 1930: 48.

“Cyprogenia in Cypern” Seidlitz, 1888: Gattungen: 60; Seidlitz, 1889: Gattungen: 60.

Alfieriella denticulata Ratti, 1976: 120, figg. 1 e 5 (designazione del Lectotypus); Leschen, 1996: 610, figg. 14 e 196 (sub denticulata), fig. 28 (sub denticollis, per lapsus calami); Johnson et al., 2007: 530.
Materiale esaminato (14 es.):

Cipro. [Cipro, leg. E. Truqui, coll. Baudi] "det. E. Ratti 1974", Lectotypus 우 e 2 Paralectotypi 우우 (MRSNT, coll. Baudi); "Cypern, leg. Baudi, coll. Reitter" (con etichetta autografa di Baudi: "Cyprogenia denticulata Baudi" e le aggiunte "Cypern, Typ., Baudi" di mano di Reitter), "Paratypus Cyprogenia denticulata Baudi 1870", 1 Paralectotypus o (HMNH); "Cypern", "denticulata B.di Coll. Reitter", 2 우 (HMNH); "Larnaka Saline", "denticulata B.di Coll. Reitter", 1 \% (HMNH); “Agh. Epictitos”, toponimo identificabile con la località Agios Epiktitos sulla costa settentrionale di Cipro, a $6 \mathrm{~km}$ ad est di Keryneia e a $15 \mathrm{~km}$ a nord di Lefkosia (= Nicosia), senza altre indicazioni, 1 o (MRSNT); "Spatarikó", toponimo identificabile con la località Spathariko sulla costa orientale di Cipro, a circa $12 \mathrm{~km}$ a Nord di Ammochostos (= Famagosta), senza altre indicazioni, 2 우우 (MRSNT, MSNG).

Israele. "Syrien, Kaifa, Reitter”, “denticulata B.di Coll. Reitter", 1 đo e 2 우, una delle quali con etichetta "det. R. Leschen" (HMNH); "Syria", "Cyphogenia [sic !] denticulata Baudi, Syria, det. Reitter", "c.[oll.] Epp[e]lsh.[eim], Steind.[achner] d.[edit]", 1 ô (NHMW).

Johnson et al. (2007) citano la specie di Cipro, Libano e Siria; io non ho esaminato materiali del Libano, dove la presenza del taxon in questione è verisimile, ma ritengo utile ricordare che a fine Ottocento con "Syria" si intendeva in genere un'area ben più ampia dell'attuale estensione della nazione siriana; in particolare alcuni degli esemplari citati di "Siria" da Reitter (1882 e 1891) provengono in effetti da Kaifa (= Haifa), che attualmente rientra nel territorio di Israele.

Alfieriella naxiana (Reitter, 1884)

(Figg. 3-4, 6-15, 21-22)

Cyprogenia naxiana Reitter, 1884: 65; Seidlitz, 1888: Gattungen: 60; Seidlitz, 1889: Gattungen: 60; Reitter, 1891: 197; Jakobson, 1915: 906; Hetschko, 1930: 49. Cyprogenia naxia Bedel, 1897: 35 (emendamento non giustificato).

Alfieriella naxiana Ratti, 1976: 119, fig. 3; Johnson et al., 2007: 530.

Secondo quanto affermato nella diagnosi originale del 1884, Reitter descrisse la specie sulla base di un unico esemplare (Holotypus) della propria collezione, raccolto nell'Isola di Naxos da Theobald Jo- 
hannes Krüper (1829-1917) e donatogli da Wilhelm Scriba (1817-1898).

Materiale ESAminato (114 es.) o COMUNiCATOMI DA Angelini (73 es.):

Grecia."Naxos", "Cyprogenia naxiana m. Naxos", "proth.[orax] ang.[ulis] post.[icis] acutissimis" (autografi di Reitter), "Naxos, Coll. Reitter", "Monotypus Cyprogenia naxiana Reitter 1884", Holotypus $\widehat{\partial}$ (HNHM); "Leonis, Athen", "naxiana Reitt., Graecia",

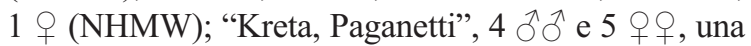
delle quali etichettata "denticincta [sic !] det. Obenberger" (NHMW).

Italia. Calabria, Aspromonte - Africo (RC), prato, $50 \mathrm{~m}$, 17.XI.1993, G. Sabella, 11 ô ô e 10 우 (MSNG), 10 추 e 9 웅 (CFA-MZUF); id., id., F. Angelini: 14.IV.1997, 1 q (MSNG); 7.XI.1997, 5 es. (MSNG), 5 es. (CFA-MZUF); 11.III.2000, 33 es. (CFA-MZUF); 27.IV.2002, 4 es. (CFA-MZUF); 29.V.2002, 6 es. (CFAMZUF); 23.I.2003, 1 o e 3 우우 (MSNG), 3 es. (CFAMZUF), 8 es. (CFA); Africo (RC), dint. foce Fiumara La Verde, vaglio detriti, 24.I.2003, F. Angelini, 5 es. (CFA); Africo (RC), strada per Samo, prato, $100 \mathrm{~m}$, 8.XI.1997, F. Angelini, 15 es. (MSNG), 10 es. (CFAMZUF); Caraffa del Bianco (RC), prato, $200 \mathrm{~m}$, 27.IV.2002, F. Angelini, 1 es. (CFA-MZUF); Ferruzzano (RC), prato, 200 m, 22.I.2003, F. Angelini, 2 es. (CFA-MZUF); Samo (RC), Fiumara Santa Venere, lentisco, 7.XI.1997, F. Angelini, 2 수 $\widehat{x}$ e 2 우우 (MSNG); Samo (RC), lentisco, 12.III.2000, F. Angelini, 4 es. (CFA-MZUF); San Luca (RC), Fiumara Santa Venere, prato, 16.XI.1993, G. Sabella, 1 ㅅ e 2 우 (MSNG), 2 es. (CFA-MZUF); San Luca dint. (RC), prato, F. Angelini: 8.XI.1997, 5 es. (MSNG), 4 es. (CFA-MZUF); 22.I.2003, 3 es. (CFA-MZUF).

Sicilia - Carlentini (SR), III.1965, A. Focarile, 1 ภ (MSNG); Melilli (SR), 100 m, 5.IV.1997, F. Angelini, 1 (MSNG); Riserva Naturale Vendicari (SR), olivo + lentisco, 6.IV.1997, F. Angelini, 1 ô e 1 q (MSNG); id., prato, 15.III.2000, F. Angelini, 1 ते e 1 क (MSNG), 2 es. (CFA); Pachino (SR), III.1913, A. Dodero, "Cyprogenia naxiana Reitt. var." (det. Dodero), 1 ㅇ (MSNG, coll. Dodero).

A Ratti (1976) non era stata concessa la possibilità di risolvere il problema del nome denticincta, con cui Jan Obenberger identificò a suo tempo gli esemplari di Creta raccolti da Gustav Paganetti-Hummler e conservati nel Museo di Vienna; restava infatti l'incertezza sul fatto che lo studioso ceco avesse voluto veramente separare "denticincta" come specie a sé o che invece si fosse trattato di un suo semplice lapsus calami per "denticulata". L'incertezza interpretativa resta, ma viene ora superata per il fatto che gli individui cretesi, anche se hanno un aspetto un po' più tozzo, col pronoto un po' più largo, rispetto a quelli italiani (Figg. 21-22), per la morfologia edeagica (Figg. 11-12) sono attribuibili a naxiana, il che è anche più congruente con la collocazione geografica di Creta.

La distribuzione accertata della specie copre quindi la Grecia (Isole di Naxos e di Creta ed area continentale ateniese) e, per quanto concerne l'Italia, solo la Calabria meridionale (provincia di Reggio Calabria) e la Sicilia sudorientale (provincia di Siracusa).

Per favorire il riconoscimento di $A$. naxiana a livello italiano se ne fornisce qui di seguito una sintetica ridescrizione.

Colore rossastro-testaceo. Superficie dorsale alutacea, con punteggiatura assai fitta, debole ed irregolare e con pubescenza corta, rada e coricata.

Lunghezza media: $\mathrm{mm} \mathrm{1,25}$; a tal proposito si osserva una discreta variabilità dimensionale: misurando 40 esemplari di Africo (21 $\partial^{\lambda}{ }^{\lambda}$ e 19 q $\odot$ ), raccolti lo stesso giorno nella stessa località, si è evidenziato che la lunghezza dei $\widehat{\partial} \hat{\sigma}$ varia da 1,12 a $1,41 \mathrm{~mm}$, mentre quella delle $ㅇ+q$ è compresa tra gli estremi di 1,08 e $1,42 \mathrm{~mm}$.

Capo un po' più lungo $(\mathrm{mm} 0,26)$ che largo $(\mathrm{mm}$ $0,23)$, nettamente più stretto del pronoto. Occhi composti da una ventina di ommatidi. Guance ad angolo retto. Antenne di 10 articoli, col $1^{\circ}$ assai ingrossato, il $2^{\circ}$ robusto e gli articoli 3-9 più esili, appena più lunghi che larghi; l'ultimo dilatato a clava, lungo quanto i 4 precedenti considerati insieme (Fig. 6).

Pronoto un po' più largo $(\mathrm{mm} 0,38)$ che lungo ( $\mathrm{mm} 0,35)$, con profilo laterale leggermente angoloso dietro la metà, ove raggiunge la massima larghezza, e con angoli posteriori ben marcati.

Elitre circa 1,5 volte più lunghe $(\mathrm{mm} 0,73)$ che larghe $(\mathrm{mm} 0,35)$, più larghe del pronoto già alla base e con la massima larghezza dietro la metà. Omeri dentati. Strie elitrali indistinte. Ali assenti.

Zampe esili; tarsi tutti pentameri, con i primi 4 articoli appena più lunghi che larghi ed uguali tra loro; l'ultimo quasi 4 volte più lungo che largo, lungo quanto i 3 precedenti presi insieme (Fig. 7).

Edeago come da Figg. 11-15, molto voluminoso ed occupante circa i 7/10 della lunghezza dell'addome; tegmen con parameri liberi e con due apofisi apicali chitinizzate a forma di martelletto; bracci del tegmen più o meno lungamente fusi nella porzione basale; lobo me- 
diano con apice arrotondato; strutture endofalliche poco sclerificate. Spiculum gastrale come da Fig. 8 . 9 e 10.

Spermateca e genitali femminili come da Figg.

Differenze sessuali: i maschi presentano il $1^{\circ}$ sternite visibile leggermente impresso nella porzione mediana e l'ultimo con una evidente depressione mediana rotondeggiante; entrambi gli sterniti sono semplici nella femmina. Sex ratio prossima a 1:1.

Secondo quanto comunicatomi da Angelini (in litt.), ad Africo la specie è stata raccolta sul pendio di una collinetta esposta ad est, a poche centinaia di metri dal mare, in prati su terreni argillosi, vagliando l'erba presente attorno a pietre e il sottostante suolo, in mesi autunnali ed invernali, in periodi in cui il terreno era molto umido a seguito di abbondanti piogge; nel medesimo sito la specie risultava molto più rara o assente tra marzo e maggio con terreno nettamente più asciutto; alcuni esemplari sono stati reperiti pure vagliando il detrito alla base di lentischi; nelle località di rinvenimento le altitudini sono comprese tra 0 e 200 metri. A giudicare anche dall'analisi di un contenuto intestinale, il regime alimentare degli esemplari parrebbe saprofago.

Alfieriella rabinovitchi Wittmer, 1935

(Figg. 2, 18-19)

Alfieriella rabinovitchi Wittmer, 1935: 131, figg. 1-3 [la fig. 2 è stampata capovolta e va ruotata di $180^{\circ}$ ]; Ratti, 1976: 120, figg. 2 e 4; Alfieri, 1976: 126 (sub rebinovitchi, per lapsus calami); Leschen, 1996: 610; Johnson et al., 2007: 530; El-Torkey et al., 2007: 88.

Materiale esaminato (2 es.):

Egitto. "Egypte, Ikingy Mariout, 29.8.1934, A. Rabinovitch", Holotypus $q$ e 1 Paratypus $\widehat{\overbrace{}}$ (MSNM). L'Holotypus in realtà è etichettato "Alfieriella decemarticulata Wittm.", nome in litteris assegnato da Wittmer alla nuova specie, prima di pubblicarla dedicandola al raccoglitore A. Rabinovitch.

Quattro paratypi della specie, secondo El-Torkey et al. (2007), sono presenti nella Collection of Ministry of Agriculture, Plant Protection Research Institute (Dokki-Giza, Egypt), etichettati "King Mariout, 23.VIII.1934, Rabinovitch" (1 "cotype") e "King Mariout, 29.VIII.1934, Rabinovitch" (3 "cotypes").

Altri paratypi sono presenti nella collezione di Anastase Alfieri, conservata nel Department of Entomology, Faculty of Agriculture, Al-Azhar University
(Nasr City, Cairo, Egypt); Alfieri (1976) cita come esemplari tipici quelli raccolti in aprile ed agosto ad Abu Mina, località in effetti posta un po' più a sud di King Mariout che è stata indicata come "locus classicus" da Wittmer (1935).

I reperti della specie al momento sembrano limitati all'area mediterranea a sudovest di Alessandria, nella zona dove oggi sorge l'Aeroporto di Borg El Arab.

Le località accertate per le specie presenti nel Mediterraneo centro-orientale sono cartografate nelle Figg. 23 e 24; il corotipo di riferimento per il genere potrebbe essere di tipo centrasiatico-mediterraneo con estensione himalayana, anche se è alquanto azzardato definirlo con esattezza in mancanza di una revisione degli elementi orientali (Uzbekistan, Afghanistan, Sikkim); A. naxiana costituisce comunque un bell'esempio di distribuzione transionica.

Le tre specie mediterranee possono distinguersi in base ai caratteri sintetizzati nella tabella che segue, essenzialmente utilizzando la morfologia dei parameri; infatti, mentre le altre componenti dell'edeago presentano un aspetto sostanzialmente omogeneo nei diversi taxa, sono i parameri ad offrire i migliori caratteri diagnostici. Essi sono appiattiti e ricoperti da numerose setole, in genere di maggiori dimensioni lungo i bordi e più brevi sulla superficie dorsale; in tutte le specie sono presenti due setole molto lunghe alla base di ogni paramero, ben visibili soprattutto se si disarticola il tegmen dal lobo mediano; al di là della chetotassi comunque il semplice profilo dei pezzi consente un'agevole distinzione, poiché i parameri di naxiana, ovalari, hanno una maggiore lunghezza ( $\mathrm{mm} 0,083-0,091)$ rispetto a quelli

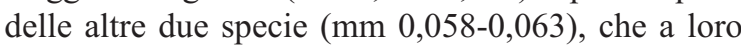
volta si presentano arrotondati in denticulata e rastremati all'apice in rabinovitchi (si confrontino in particolare le Figg. 14, 16 e 18).

1) Lati del pronoto a profilo leggermente angoloso (Figg. 3, 4, 21, 22). Parameri più allungati: $\mathrm{mm}$ 0,083-0,091 (Figg. 11-14). Grecia e Italia. naxiana

- Lati del pronoto a profilo arrotondato (Figg. 1, 2, 20). Parameri più brevi: mm 0,058-0,063 (Figg. 16 e 18)...

.2

2) Parameri come da Fig. 16 (lungh. mm 0,058). Cipro, Libano, Israele denticulata

- Parameri come da Fig. 18 (lungh. mm 0,063). Egitto settentrionale. rabinovitchi 

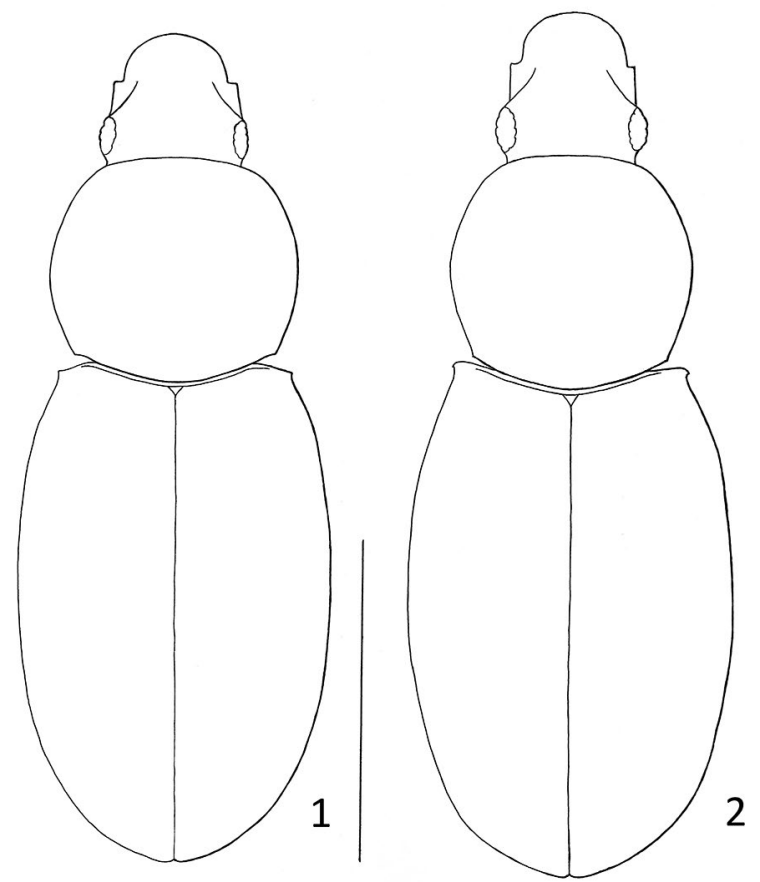

Figg. 1-2. Profilo schematico del corpo di Alfieriella. $1-A$. denticulata, Lectotypus $\rightarrow$ di Cipro; $2-A$. rabinovitchi, Holotypus $q$ di King Mariout. Scala $=$ mm 0,5.
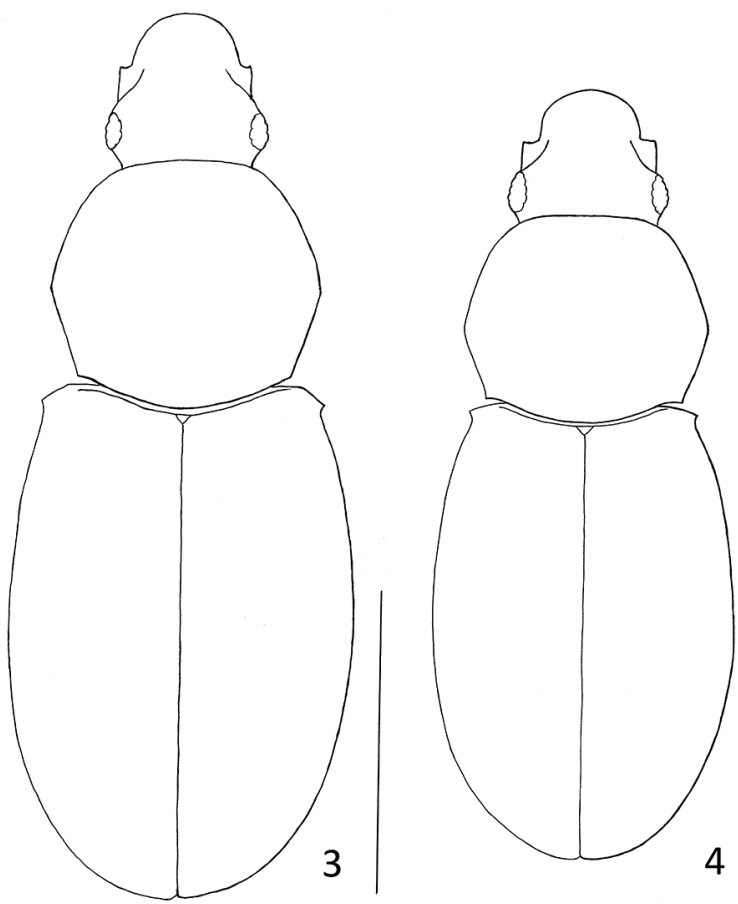

Figg. 3-4. Profilo schematico del corpo di Alfieriella naxiana. 3 - Holotypus $\widehat{o}$ di Naxos; $2-\hat{o}$ di Carlentini. Scala $=\mathrm{mm} 0,5$.

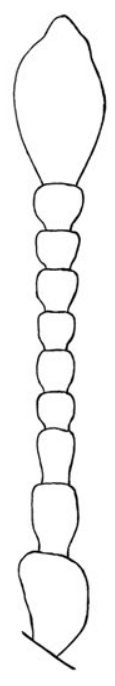

5

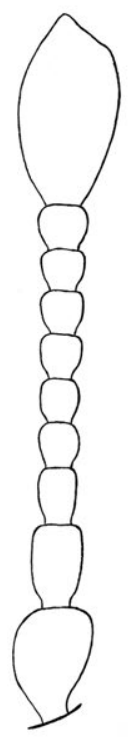

6

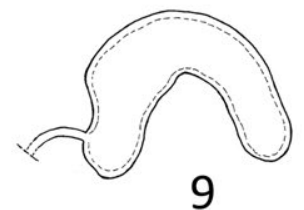

9

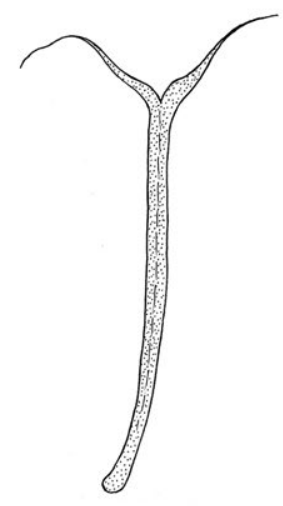

a

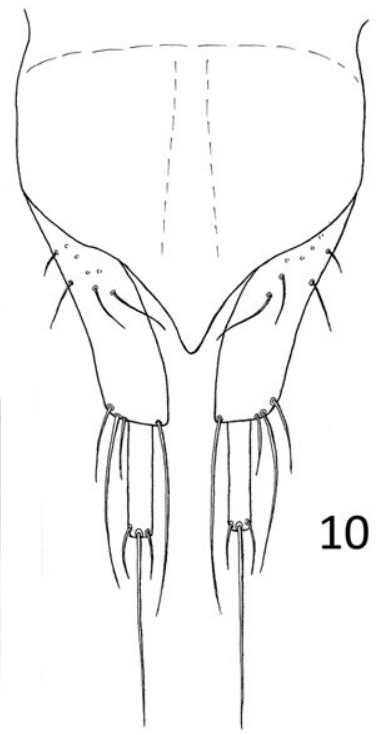

8

Figg. 5-10. 5 - Antenna di Alfieriella denticulata, Lectotypus $\varnothing$ di Cipro; 6 - antenna di A. naxiana, ô di Carlentini; 7 metatarso di A. naxiana, $\widehat{\partial}$ di Carlentini; 8 - spiculum gastrale di A. naxiana, $\widehat{\partial}$ di Carlentini; 9 - spermateca di A. naxiana, +9 di Africo; $10-$ ultimi uriti di $A$. naxiana, + di Africo. Scala $=$ mm 0,1 (a per figg. 5-7; b per figg. 8-10). 

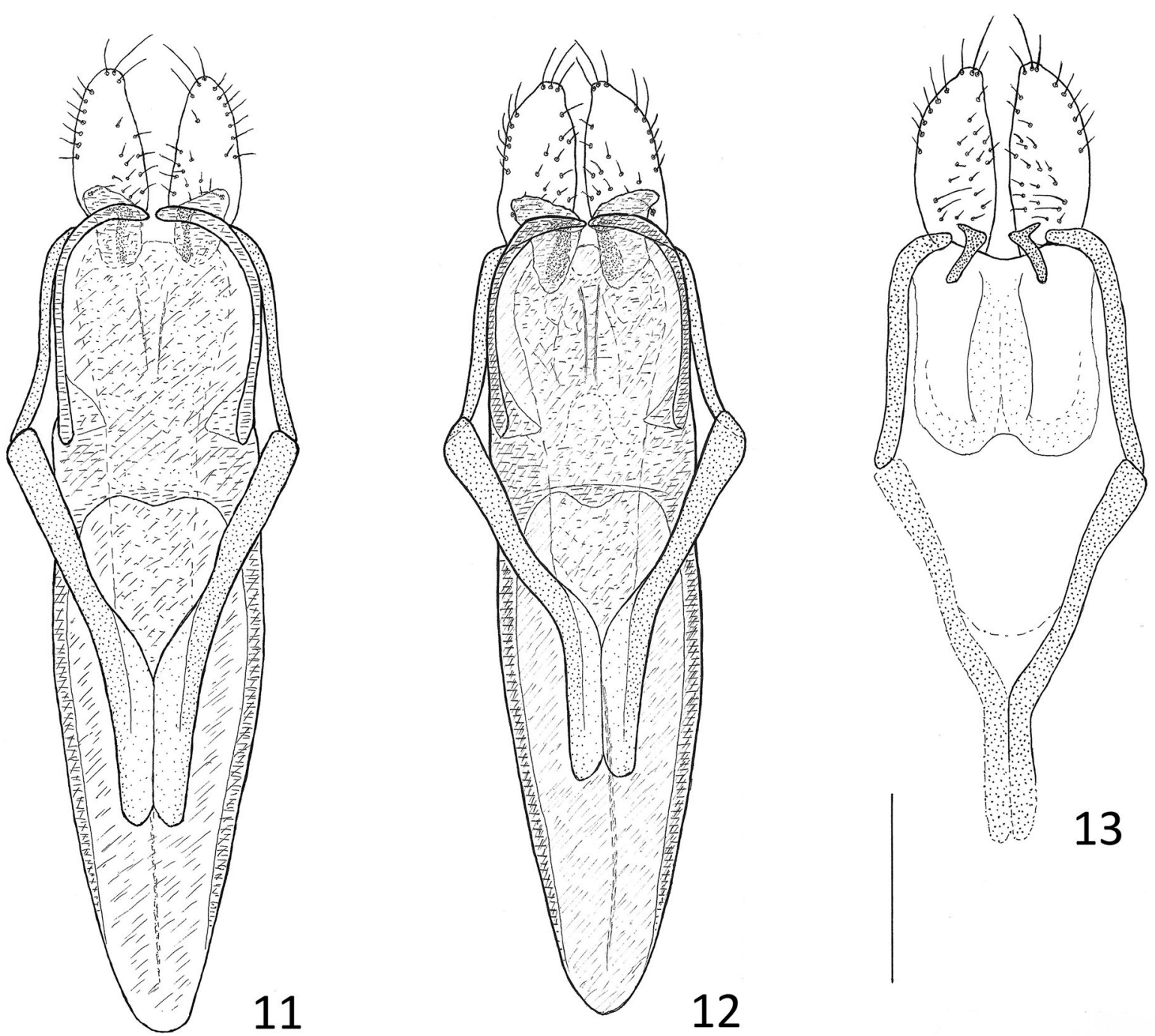

Figg. 11-13. Edeagi di Alfieriella naxiana: 11 - ồ di Creta (non disarticolato); 12 - ô di San Luca (non disarticolato); 13 - Holotypus $\widehat{\partial}$ di Naxos (il solo tegmen). Scala $=\mathrm{mm} 0,1$. 

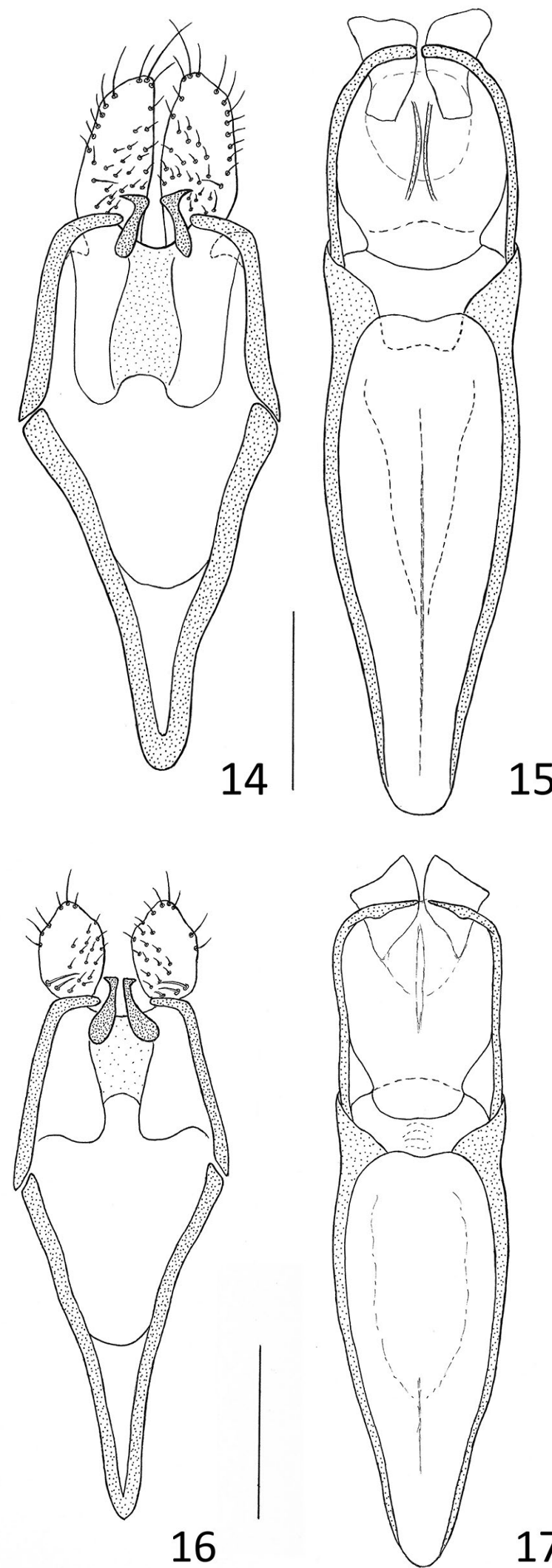

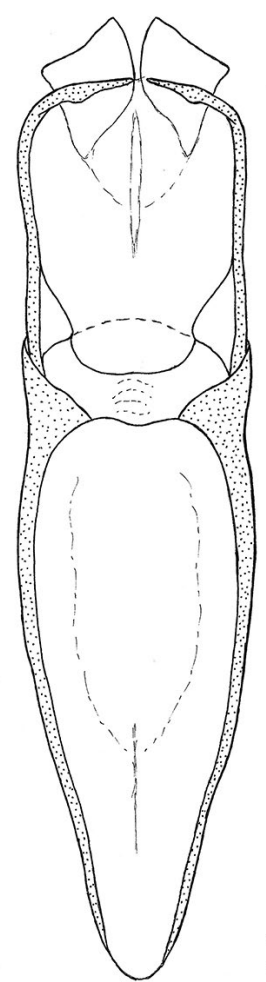

17
Figg. 14-15. Edeago disarticolato di Alfieriella naxiana, $\hat{\jmath}$ di Carlentini. Scala $=\mathrm{mm} 0,1$.

Figg. 16-17. Edeago disarticolato di Alfieriella denticulata, $\delta$ di Agios Epiktitos. Scala $=\mathrm{mm} \mathrm{0,1.}$ 

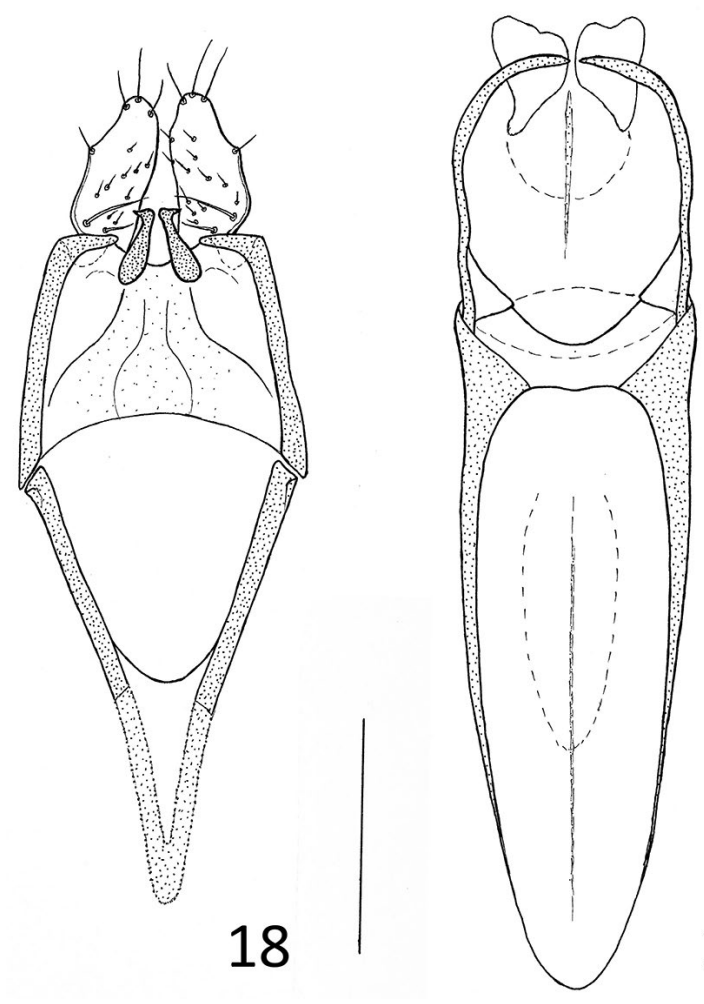

19 Figg. 18-19. Edeago disarticolato di Alfieriella rabinovitchi, Paratypus ${ }^{\widehat{D}}$ di King Mariout. Scala $=\mathrm{mm} 0,1$.
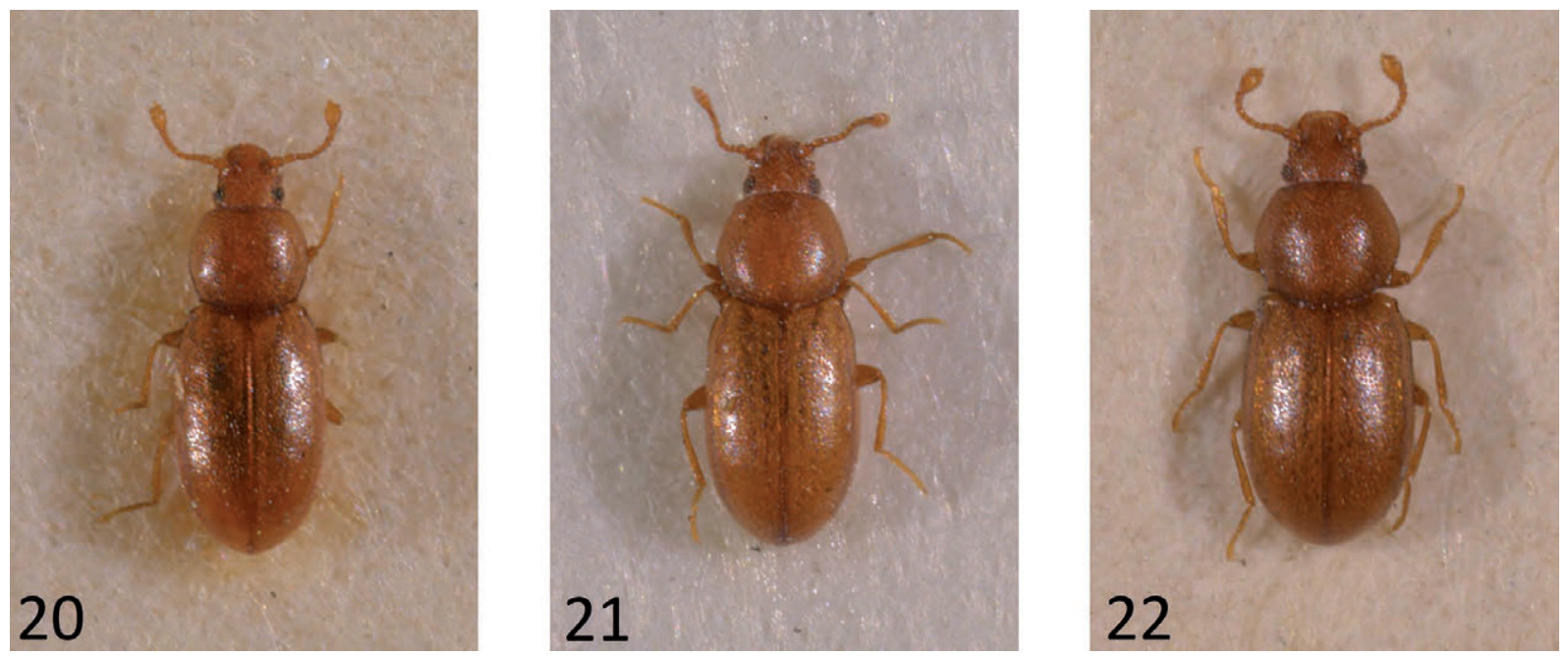

Figg. 20-22. Habitus di Alfieriella: 20 - A. denticulata, Lectotypus $\bigcirc$ di Cipro; $21-A$. naxiana, ô di Carlentini; $22-A$. naxiana, ô di Creta. 


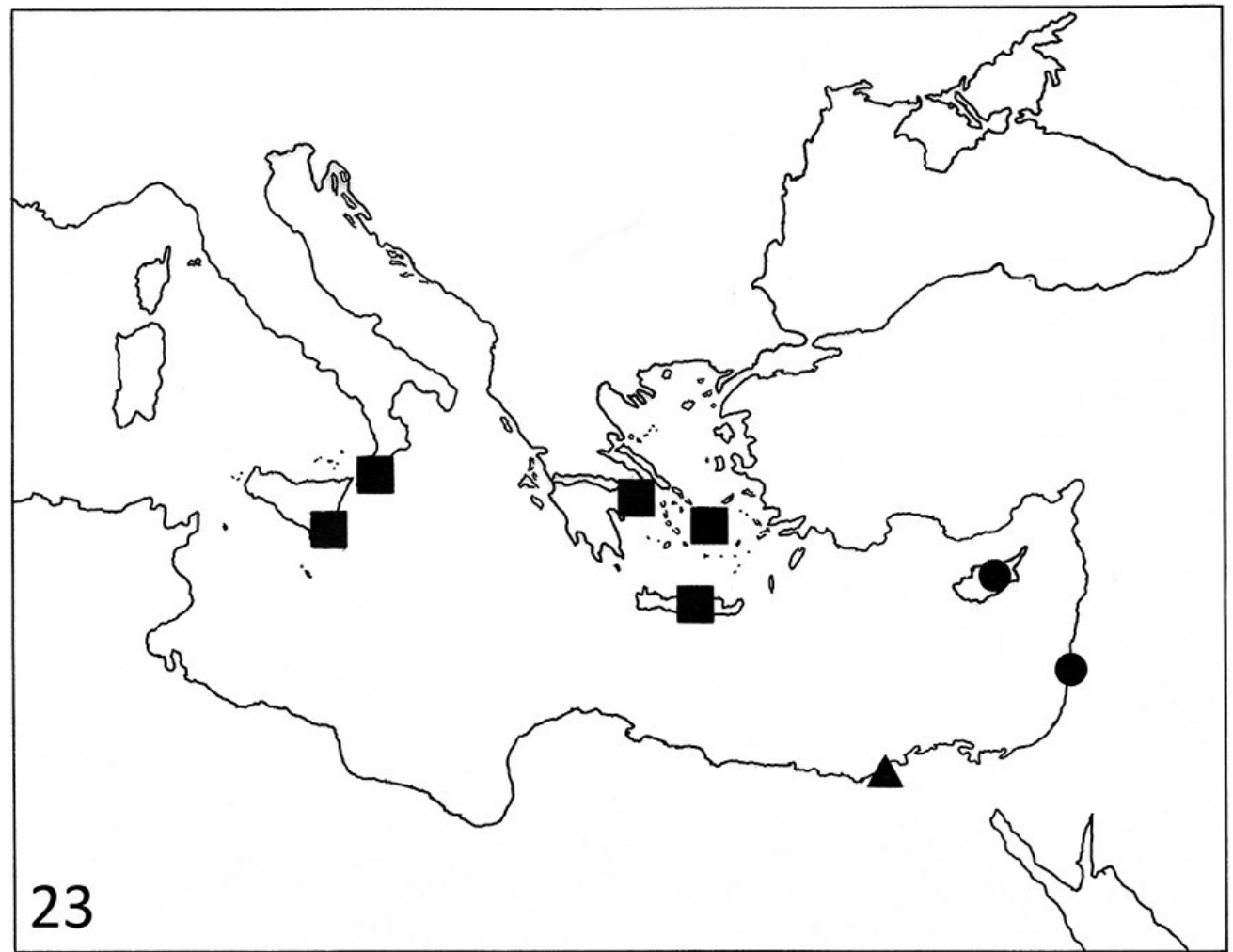

Fig. 23. Cartina di distribuzione generale delle specie mediterranee di Alfieriella . Cerchio $=$ A . denticulata; quadrato $=A$. naxiana , triangolo $=A$. rabinovitchi .

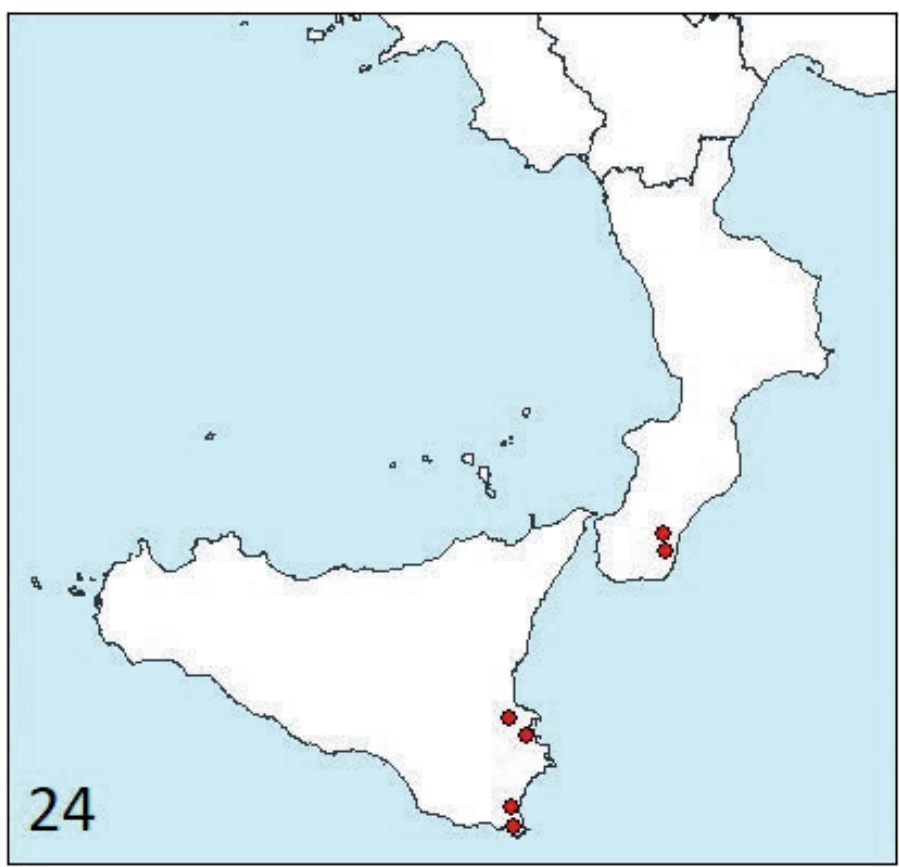

Fig. 24. Cartina particolareggiata dei reperti italiani di Alfieriella naxiana. 


\section{BIBLIOGRAFIA}

Alfieri A., 1976 - The Coleoptera of Egypt. Mémoires de la Société entomologique d’Egypte, Cairo, 5: XVI + 1-361, 1 fig., 4 tavv. Angelini F., 1995 - Cryptophagidae e Languriidae. In: Angelini F., Audisio P., De Biase A., Poggi R., Ratti E., Zampetti M.F., 1995 - Coleoptera Polyphaga X (Clavicornia I). In: Mineldi A., Ruffo S., LA Posta S. (eds.), Checklist delle specie della fauna italiana, 55. Ed. Calderini, Bologna, $20 \mathrm{pp}$.

BAUdi DI SElve F., 1870 - Coleopterorum messis in insula Cypro et Asia minore ab Eugenio Truqui congregatae recensitio: de Europaeis notis quibusdam additis. Pars tertia. Berliner entomologische Zeitschrift, Berlin, 14: 49-90.

BEDEL L., 1897 - Recherches synonymiques et rectificatives. L'Abeille, Paris, 29: 35-36.

BehURA T., 1992 - Classification and taxonomic studies on Indian Cryptophagidae (Coleoptera: Insecta) - Tesi di laurea, Utkal University, Vani Vihar, Bhubaneswar (India), 317 pp., 321 figg.; https://shodhganga.inflibnet.ac.in (accesso: 20.II.2020).

Bouchard P., Bousquet Y., Davies A.E., Alonso-Zarazaga M.A., Lawrence J.F., Lyal C.H.C., Newton A.F., Reid C.A.M., Schmitt M., ŚliPIŃSKi S.A., SMITH A.B.T., 2011 - Family-group names in Coleoptera (Insecta). ZooKeys, Sofia, 88: 1-972.

Crowson R.A., 1980 - On amphipolar distribution patterns in some cool climate groups of Coleoptera. Entomologia generalis, Stuttgart-New York, 6 (2-4): 281-292, 2 figg., 6 cartine.

El-Torkey A.M., El-Gharbawy A.A., Abdel-Dayem M.S., 2007 - A review of silken fungus beetles in Egypt (Coleoptera: Cryptophagidae). Bulletin of the entomological Society of Egypt, Cairo, 84: 85-101, 26 figg.

Ganglbauer L., 1899 - Die Käfer von Mitteleuropa. Die Käfer der österreichisch-ungarischen Monarchie, Deutschlands, der Schweiz, sowie des französischen und italienischen Alpengebietes. Band III. Ed. C. Gerold's Sohn, Wien, pp. III + 1046.

Hetschко A., 1930 - Coleopterorum Catalogus auspiciis et auxilio W. Junk editus a S. Schenkling. Pars 109. Cucujidae, Thorictidae (Suppl.), Cossyphodidae (Suppl.). Ed. Junk, Berlin, 122 pp.

ICZN (International Commission on Zoological Nomenclature), 1999 - International Code of Zoological Nomenclature. Fourth edition. International Trust for Zoological Nomenclature, London, xxix $+306 \mathrm{pp}$.

JAKOBSON G.G., 1915 (Vypusk 11, pp. 865-1024) - Zhuki Rossi i zapadnoy Evropy. Rukovodstvo k opredelenyu Zhukov. Devrien, St. Petersburg, 1905-1915, 1024 pp., 83 pl.

Johnson C., Otero J.C., Leschen R.A.B., 2007 - Cryptophagidae (pp. 513-531) - In: Löbl I., Smetana A., Catalogue of Palaearctic Coleoptera Vol. 4. Apollo Books, Stentrup, 935 pp.

LESCHEN R.A.B., 1996 - Phylogeny and revision of the genera of Cryptophagidae (Coleoptera: Cucujoidea). University of Kansas Science Bulletin, Lawrence, 55 (15): 549-634, 209 figg., 1 tab.

LESCHEN R.A.B., 1999 - Origins of symbiosis: phylogenetic patterns of social insect inquilinism in Cryptophagidae (Coleoptera: Cucujoidea). University of Kansas Natural History Museum Special Publications, Lawrence, 24: 85-101, 5 figg., 1 tab.

Otero C., 2013 - Fauna Europaea: Cryptophagidae. In: Audisio P., Fauna Europaea: Coleoptera Polyphaga Cucujiformia; https://fauna-eu.org (accesso: 18.I.2020).

RATti E., 1976 - Nota sul genere Alfieriella Wittmer, 1935 ( = Cyprogenia Baudi, 1870, nec Agassiz, 1852) syn. n., con discussione sulla sua posizione sistematica. Bollettino della Società entomologica italiana, Genova, 108 (5-7): 118-122, 5 figg.

ReitTer E., 1882 - Bestimmungs-Tabellen der europäischen Coleopteren. VI. Enthaltend die Familien: Colydiidae, Rhysodidae, Trogositidae. Verhandlungen des naturforschenden Vereines in Brünn, 20 (1881): 113-149.

ReitTer E., 1884 - In: Brenske E., ReitTER E. - Neuer Beitrag zur Käferfauna Griechenlands. Deutsche entomologische Zeitschrift, Berlin, 28 (1): 17-100, tavv. I e II.

REITTER E., 1891 - Zweiter Beitrag zur Coleopteren-Fauna des russischen Reiches. Wiener entomologische Zeitung, Wien, 10 (6): 195-199.

ReITter E., 1922 - Bestimmungs-Tabellen der europaeischen Coleopteren. VI. Enthaltend die Familien: Colydiidae, Rhysodidae, Ostomidae. Zweite, gänzlich umgearbeitete und auf die palaearktische Fauna ausgedehnte Auflage. Ed. Reitter, Troppau, 73 pp.

SEIdLITZ G., 1888 (pp. xli-xlviii + 17-80 Gattungen + 97-336 Arten) - Fauna Baltica. Die Kaefer (Coleoptera) der deutschen Ostseeprovinzen Russlands. Zweite neu bearbeitete Auflage. Hartungsche Verlagsdruckerei, Königsberg, 1887-1891, lvi + 192 (Gattungen) + 914 (Arten) pp., 1 Taf.

SEIDLITZ G., 1889 (pp. xli-lvi + 49-128 Gattungen + 241-544 Arten) - Fauna Transsylvanica. Die Kaefer (Coleoptera) Siebenbürgens. Hartungsche Verlagsdruckerei, Königsberg, 1888-1891, 10 + lvi + 192 (Gattungen) + 818 (Arten) pp., 1 Taf.

ŚLIPIŃSKI S.A., 1983 - A review of the Ethiopian species of Passandra Dalman, with notes on the constitution of the Cucujidae (Coleoptera). Polskie Pismo entomologiczne, Wrocław, 53: 77-105, 19 figg.

STRAND E., 1936 - Miscellanea nomenclatoria zoologica et paleontologica. Folia zoologica et hydrobiologica, Riga, 9: 167-170.

WitTMER W., 1935 - Ein neues Genus aus der Familie der Cucujidae (Coleoptera - Clavicornia). Bulletin de la Société royale entomologique d'Egypte, Cairo, 19: 129-131, 3 figg. 